\title{
In vitro predegeneration of peripheral nerve; the effect of predegeneration period on rat Schwann cell cultures
}

\author{
PETER TOMKO, LUCIA SLOVINSKÁ and IVO VANICKÝ \\ Department of Regenerative Medicine and Cell Therapy, Institute of Neurobiology, \\ Slovak Academy of Sciences, 04001 Košice, Slovakia
}

Received September 22, 2017; Accepted January 26, 2018

DOI: $10.3892 /$ etm.2018.7021

\begin{abstract}
Peripheral nerve predegeneration has been used as a tool to improve the in vitro cultivation of Schwann cells. The process of predegeneration may be accomplished either in vivo or in vitro. In previously published studies, various predegeneration periods were used, ranging from a few days until up to 5 weeks. The present study systematically evaluated the effect of various durations of in vitro predegeneration on the efficacy of Schwann cell cultivation. The sciatic nerves of adult Wistar rats were harvested and the explanted nerve pieces were maintained in the predegeneration medium for different predegeneration periods. In group A, the dissociation was performed immediately after harvesting. In groups B, C and D, the predegeneration periods were 2, 4 and 6 weeks, respectively. During the predegeneration period, the tissue pieces were repeatedly transferred into new dishes. Afterwards, the nerve tissue was enzymatically dissociated and the cells were seeded onto a six-well culture plate at a defined density. After 3-4 days of incubation, the cultures were passaged by means of the cold jet technique and the cell cultivation was continued for another 21 days. It was observed that the cell cultures in groups A and B were rapidly overgrown by fibroblasts. In group C, numerous wells contained a highly enriched Schwann cell population that had formed a typical monolayer, but in a fraction of the dishes, cultures were debased by fibroblast overgrowth. In group D, all of the cultures had enriched Schwann cell populations. In the experiments of the present study, the positive effect of predegeneration was observed only when the predegeneration periods lasted for 4 weeks or longer. It was
\end{abstract}

Correspondence to: Dr Peter Tomko, Department of Regenerative Medicine and Cell Therapy, Institute of Neurobiology, Slovak Academy of Sciences, 4 Šoltésovej, 04001 Košice, Slovakia E-mail: tomko@saske.sk

Abbreviations: ATB, antibiotics; CJ, cold jet; DMEM, Dulbecco's modified Eagle's medium; NGS, normal goat serum; SCM, SC medium; SCs, Schwann cells

Key words: Schwann cells, predegeneration period, cultivation, in vitro, peripheral nerve concluded that the longer predegeneration periods activated Schwann cells and/or depleted the fibroblast proliferation capacity.

\section{Introduction}

Peripheral nerve lesions represent a serious problem in traumatic injuries $(1,2)$. Current strategies to repair the transected peripheral nerve are limited to end-to-end coaptation or autologous nerve grafting, if there are long nerve gaps after devastating injuries (3). Autologous nerve bridges are the most effective method for repairing nerve gaps. However, the use of this method is limited by additional morbidity and by restricted nerve resources. To address this issue, numerous synthetic and natural biomaterials have been tested to function as artificial nerve conduits for regrowing axons (4-6). Several experimental studies have demonstrated that the regeneration of artificial guidance channels is enhanced when the channel is seeded with Schwann cells (SCs) $(7,8)$. SCs appear to have a crucial role in the process of nerve regeneration (9). The regenerating axons do not grow through longer acellular nerve grafts if SC migration is impeded (10).

After nerve injury in adult mammals, Wallerian degeneration occurs at the distal end of the transected peripheral nerve (11). SCs depleted of their axon counterpart revert back to a non-differentiated, proliferative phenotype, become activated and start to multiply (12). This injury-induced cell plasticity has been proposed to be a transdifferentiation that generates a specialized repair cell, also termed a Büngner cell, which may be distinguished from the SCs present in the developing nerve. It has been proposed that these repair cells guide the regrowth of the injured axons and eventually remyelinate them to facilitate functional recovery of the regenerated nerve fibers.

In vitro, it is possible to generate high quantities of SCs from peripheral nerves of newborn rats $(13,14)$. However, the purification and enrichment of SCs from adult tissues is much more challenging. Several methods to isolate SCs from adult mammalian nerves have been reported. Most of the protocols published use a predegeneration step that enhances the effectivity of cultivation (15-20). The process of predegeneration appears to activate the proliferative abilities of SCs and diminish the number of fibroblasts by their migration out of the connective tissues during the predegeneration phase. However, 
different protocols use various lengths of predegeneration that vary from 1 week to $4-5$ weeks and the optimal time has remained to be determined. In order to address this question, the present study systematically assessed the effect of different predegeneration periods on SC yield in culture.

\section{Materials and methods}

Animals. A total of 8 adult female Wistar rats (body weight, 200-250 g; age, 12-16 weeks) were used in the present study (Velaz Ltd., Czechia, Prague). The animals were divided into four groups with different lengths of predegeneration periods. In group A $(n=2)$, nerves were dissociated immediately after harvesting. The in vitro predegeneration period prior to dissociation lasted for 2 weeks in group $B(n=2), 4$ weeks in group C $(n=2)$ and 6 weeks in group $D(n=2)$.

Peripheral nerve tissue harvesting. Animals were deeply anesthetized by isoflurane inhalation and killed by cervical dislocation. The skin of the hindlimbs was shaved and disinfected. Under sterile conditions, sciatic nerves on each side were removed and transferred into a Petri dish with Dulbecco's modified Eagle's medium (DMEM; BioSera, Shanghai, China) with antibiotics (ATB; $100 \mathrm{U} / \mathrm{ml}$ penicillin and $100 \mu \mathrm{g} / \mathrm{ml}$ streptomycin; Sigma-Aldrich; Merck KGaA, Darmstadt, Germany). Under the operating microscope, the epineurium was removed and the sciatic nerves were weighed. The nerves were then placed into a Petri dish with fresh cultivation medium and cut into pieces of 1-2 mm in length. Pieces from a pair of nerves were collected and placed in one well of a 6-well culture plate.

In vitro predegeneration. Nerve explants were cultivated at $37^{\circ} \mathrm{C}$ and $5 \% \mathrm{CO}_{2}$ in a predegeneration medium [SC medium (SCM)] according to Haastert-Talini (21), which contained melanocyte growth medium (PromoCell, Heidelberg, Germany) supplemented with $10 \%$ fetal bovine serum (Sigma-Aldrich; Merck KGaA), ATB, $10 \mathrm{ng} / \mathrm{ml}$ fibroblast growth factor 2 (Sigma-Aldrich; Merck KGaA), $2 \mu \mathrm{M}$ forskolin (Sigma-Aldrich; Merck KGaA) and $5 \mu \mathrm{g} / \mathrm{ml}$ bovine pituitary extract (Sigma-Aldrich; Merck KGaA). The medium was changed twice a week. Nerve fragments were transferred into a new well after 6 or 7 days, when the migrating fibroblasts had formed a near-confluent layer of cells around the nerve fragments.

Dissociation and primary plating. The nerve fragments were dissociated either immediately after harvesting (group A) or after the predegeneration periods (groups B-D).

Nerve fragments were placed into test tubes with DMEM containing 0,125\% collagenase I (Sigma-Aldrich; Merck $\mathrm{KGaA}$ ) and 1,25 U/ml dispase (Roche Diagnostics, Basel, Switzerland) for enzymatic digestion. They were incubated for $24 \mathrm{~h}$ and then mechanically triturated with micropipette, and the cell suspension was filtrated into a test tube through a $40-\mu \mathrm{m}$ cell strainer. The test tubes were then centrifuged at $235 \mathrm{x} \mathrm{g}$ for $6 \mathrm{~min}, 20^{\circ} \mathrm{C}$. The supernatant was removed, the pellet was resuspended with fresh medium and the process of centrifugation-resuspension was repeated once more. Cells in the suspension were counted and seeded into 6-well culture plates, at $\sim 1$ milion cells per well (5-7 wells from each animal). The wells were coated with laminin $(6 \mu \mathrm{g} / \mathrm{ml}$; Sigma-Aldrich; Merck KGaA) and poly-L-ortnithine hydrobromide $(1 \mathrm{mg} / \mathrm{ml}$; Sigma-Aldrich; Merck KGaA) for better adherence of SCs. Initially, cells were cultivated in SCM with addition of $1 \%$ bovine serum albumin (BSA; Sigma-Aldrich; Merck KGaA) for $24 \mathrm{~h}$. Subsequently, the medium was replaced with SCM without BSA. The medium was changed every second day. When the proliferating cells reached counflency (days 3-4), the SC population was enriched by means of the cold jet $(\mathrm{CJ})$ technique $(17,21)$.

The SCM was removed from the wells and ice-cold PBS was slowly added and aspirated 2 times to cool down the culture and loosen SCs that grow on top of the fibroblasts. Subsequently, ice-cold SCM was added in a moderate stream to detach SCs. This process was repeated once more if the SCs were not detached after the first flush with SCM. During this process, the culture was examined under the microscope in order to make sure that fibroblasts were still attached to the surface of the well and SCs were floating in the medium. The SCM containing the suspended SCs was removed, added to a test tube and centrifuged (at a speed of $235 \mathrm{x} \mathrm{g}$ for $6 \mathrm{~min}$ at $20^{\circ} \mathrm{C}$. The supernatant was removed and the pellet was resuspended with fresh SCM. The cells were then seeded onto a new coated well and the SCM was changed every second day. When the proliferating cells in culture achieved confluency (20 days), imunocytochemical staining was performed in order to distinguish SCs from other cells.

Immunocytochemistry. After removal of the medium, 4\% paraformaldehyde in 0.1 PBS was added to fix the cells at $4^{\circ} \mathrm{C}$ for $30 \mathrm{~min}$. The paraformaldehyde was then removed and wells were washed with $0,1 \mathrm{M}$ PBS twice.

A solution of $0,1 \mathrm{M}$ PBS with $0,2 \%$ Triton-X-100 (Sigma-Aldrich; Merck KGaA) and 10\% normal goat serum (NGS; Sigma-Aldrich; Merck KGaA) was added for blocking non-specific protein reactions. Cultures were incubated for $1 \mathrm{~h}$ at room temperature. The solution was then removed and substituted by a solution of the primary antibody S100 (dilution, 1:1,000; Sigma-Aldrich; Merck KgaA; cat. no. Z0311) in 0,1 M PBS with 0,2\% Triton-X-100 and 1\% NGS. After overnight incubation at $4^{\circ} \mathrm{C}$, the solution was removed and the wells were rinsed with PBS, followed by incubation with secondary antibody (fluorescein isothiocyanate-conjugated anti-rabbit immunoglobulin G; dilution, 3:1,000; DAKO, Glostrup, Denmark; cat. no. F0382) and DAPI (dilution, $1 \mu \mathrm{l}: 10 \mathrm{ml}$; Sigma-Aldrich; Merck KGaA, D9542) in 0,1 M PBS with $0,2 \%$ Triton-X-100 and $1 \%$ NGS in the dark for $1 \mathrm{~h}$ at $20^{\circ} \mathrm{C}$. Subsequently, the samples were washed with distilled water, the wells were left to dry for $2 \mathrm{~h}$ at room temperature and cells then were embedded with Mowiol (Sigma-Aldrich; Merck KgaA; cat. no. 81381) and mounted with coverslips. Prior to observation, the stained wells were kept in a refrigerator.

Cell counting. Immediately after dissociation, the cell suspension was sampled and the concentration of cells was determined by counting in a Burker chamber.

At the end of cultivation, the cell cultures were fixed and immunostained. The number of total cells and the number of SCs were determined by counting DAPI-positive nuclei and 
S100-positive cells in ten random fields per well (magnification, x10) in three different wells per animal. Counting was performed using ImageJ software, version 1.51q (National Institutes of Health, Bethesda, MD, USA).

Statistical analysis. All quantitative data are expressed as the mean \pm standard error of the mean. Statistical differences between groups without predegeneration (group A) and groups with predegeneration (groups $\mathrm{B}, \mathrm{C}$ and $\mathrm{D}$ ) were evaluated by one-way analysis of variance with post-hoc Bonferroni and Holm multiple comparisons. Calculations were made using Microsoft Excel (Microsoft Corp., Redmond, WA, USA), Online Web Statistical Calculators (www.astatsa.com) and the GraphPad online application QuickCalcs (https://www .graphpad.com/quickcalcs/). $\mathrm{P}<0.05$ was considered to indicate a statistically significant difference.

\section{Results}

General observation. During the predegeneration period, fibroblasts migrated out of the cut ends of nerve fragments (Fig. 1). Migration of fibroblasts was high during the first two weeks of in vitro predegeneration. The flattened cells were attached to the plastic and within one week, they formed a near-confluent layer. After repeated transfers of nerve fragments, the number of fibroblasts decreased. In addition, numerous clusters of small spherical cells appeared around nerve fragments. These cells were not adherent to the surface, but floated freely in the medium. Apparently, with prolonged in vitro predegeneration, the number of the migrating fibroblasts further decreased and the number of the aggregates of spherical cells in the medium increased. These spherical cells were SCs.

Group A. The nerve fragments were dissociated immediately after their isolation. After incubation, the cells adhered to the bottom of the dish and started to proliferate (Fig. 2). Within 3-4 days, the culture achieved confluency. Based on their morphology, most of the cells were fibroblasts and only few spindle-like SCs grew on the top of them. It was attempted to achieve SC enrichment by CJ application; however, it was not possible to completely remove the fibroblasts and the newly passaged cultures were again rapidly overgrown by the contaminating fibroblasts. During this process, it was obvious that the proliferation of SCs was halted when the number of fibroblasts prevailed in the growing culture (Fig. 3).

Group B. After 2 weeks of in vitro predegeneration, the time course of cellular proliferation in the culture was similar to that in group A. After dissociation and seeding, the predominant cell type were fibroblasts that rapidly outgrew the minority of $\mathrm{SCs}$ in the culture. However, within the first days in culture, the number of SCs appeared to be higher than that in group A. However, during the cultivation, fibroblasts overgrew in the culture (Fig. 4) and the CJ application had only a minimal effect on this process.

Group C. After 4 weeks of predegeneration, the early cultures contained SCs and fibroblasts, but it appeared that most of the cells had an SC-like morphology. After prolonged cultivation, the cultures developed into two types: Type 1 formed

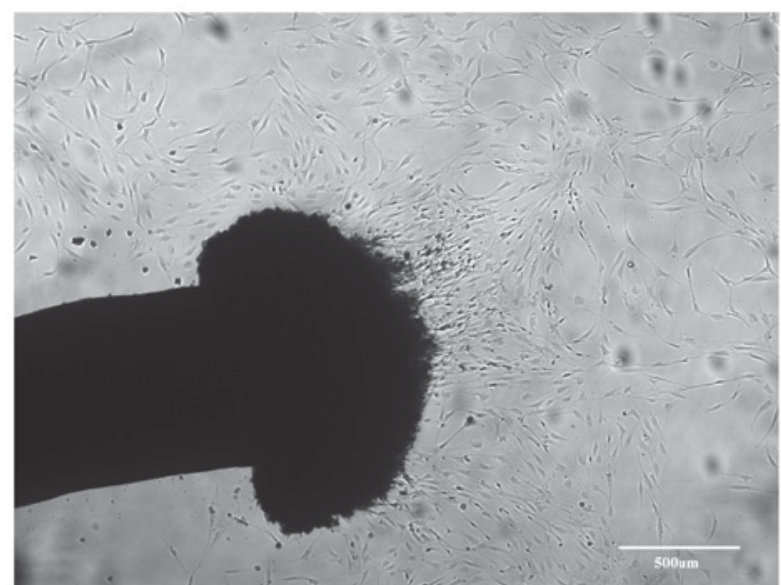

Figure 1. In vitro predegenerated nerve explant (dark structure) after 7 days in culture (scale bar, $500 \mu \mathrm{m}$ ) in group A. Outgrowing fibroblasts were attached to the surface and formed a near-confluent monolayer.

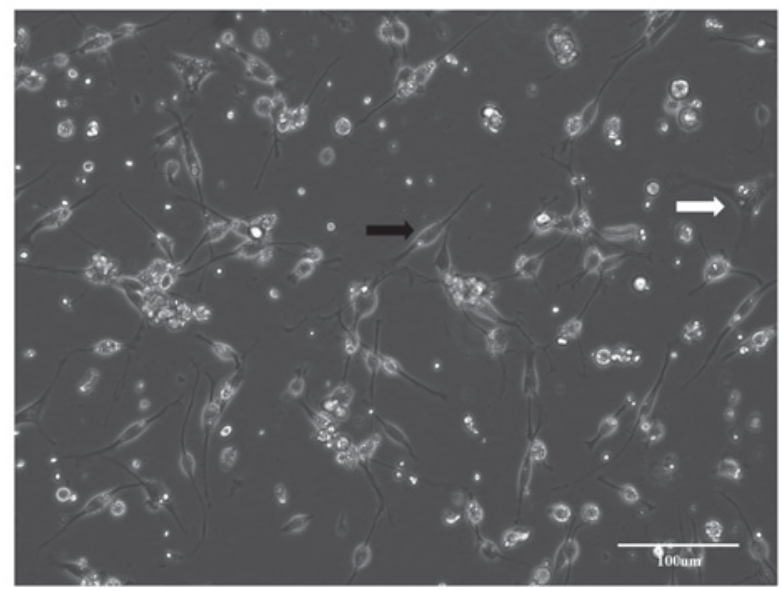

Figure 2. Cells from group D after dissociation growing on culture plates coated with laminin and poly-L-ortnithine hydrobromide (scale bar, $100 \mu \mathrm{m}$ ). After 4 days of culture, the adhered cells displayed a bi- or tripolar morphology with bright oval soma typical for Schwann cells (black arrow), or a flattened polymorphic shape characteristic of fibroblasts (white arrow).

a near-homogeneous population of SCs with a minority of scattered fibroblasts (Fig. 5) and in type 2, large areas of near-homogeneous fibroblast populations intermingled with smaller islets of proliferating SCs (Fig. 6).

Group D. The nerve fragments were predegenerated for 6 weeks. After dissociation, the cultures contained mostly SCs with only few fibroblasts. When the proliferating cells achieved confluency, they contained an almost homogeneous population of SCs and the monolayers of paralelly arranged SCs had formed typical waves (Fig. 7). Only in certain dishes, among the monolayer of SCs, small islands of homogeneous fibroblast populations were observed, particularly in the areas where the density of SCs was low.

Quantification. Cell counts after dissociation are presented in Fig. 8. The yield of cells was expressed as a number of cells per mg of wet weight of nerve after epineurium removal. Compared to the control (group A), the predegeneration 


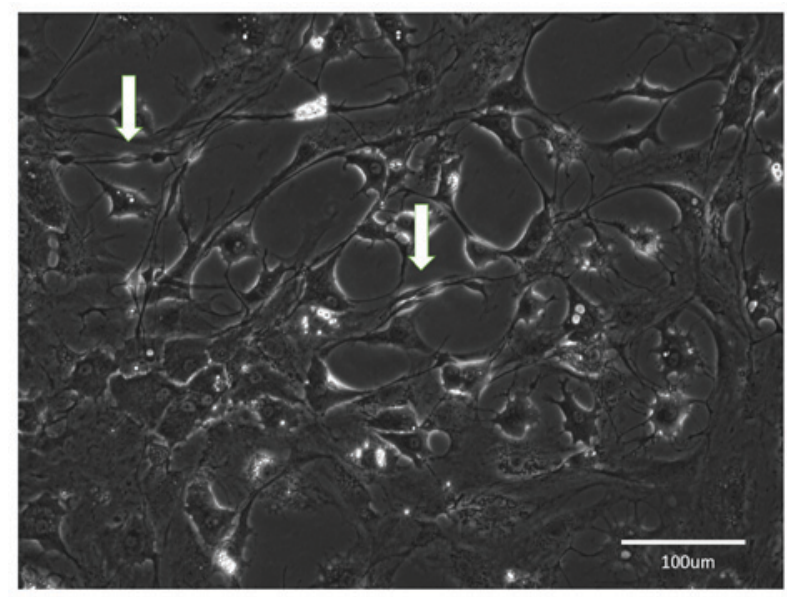

Figure 3. Group A. Debased cell culture after 2 weeks (scale bar, $100 \mu \mathrm{m}$ ). The fibroblasts prevailed and only a few cells with typical Schwann cell-like morphology (arrows) were observed.

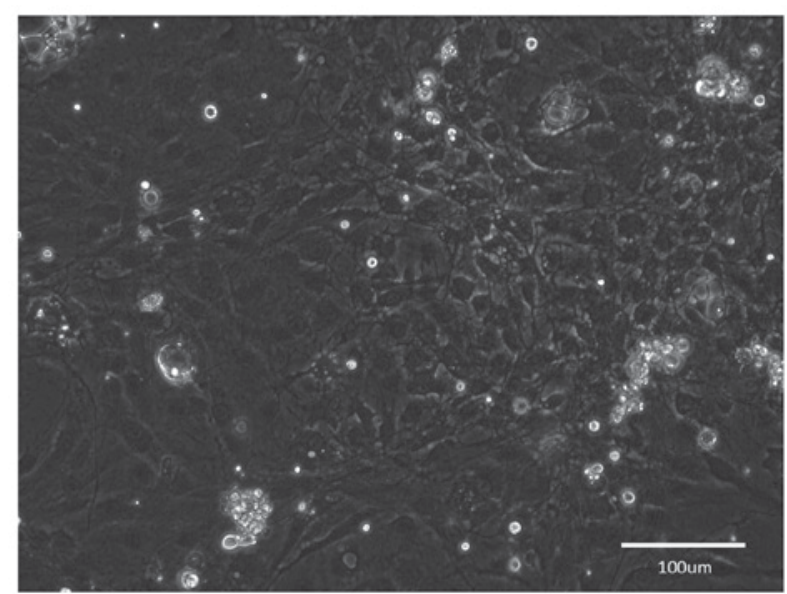

Figure 4. Group B. Debased cell culture after 2 weeks (scale bar, $100 \mu \mathrm{m}$ ) Fibroblasts formed a dense monolayer and Schwann cells were eliminated from the culture.

appeared to increase the number of dissociated cells, but the intergroup differences were relatively modest. Statisical analysis indicated significant differences between groups A and B and also between groups $\mathrm{A}$ and $\mathrm{D}$.

In immunostained cultures, the spindle-shaped cells were S100-positive (Fig. 9. The cell counts in the final cultures are presented in Fig. 10. In groups A and B, the SCs were essentially absent, and the cell numbers are therefore presented for groups C and D only.

\section{Discussion}

The predegenerated peripheral nerves form an environment that is supportive of axonal growth in the regenerating peripheral nerves and even in the central nervous system, as demonstarted by David and Aguayo (22). More recently, a concept of artificial SC-filled guidance channels has been proposed as a promising tool for bridging peripheral nerve defects $(6,23,24)$. For this purpose, production of highly enriched adult SC cultures is required. It has been reported that it is possible to obtain high quantities of enriched SCs

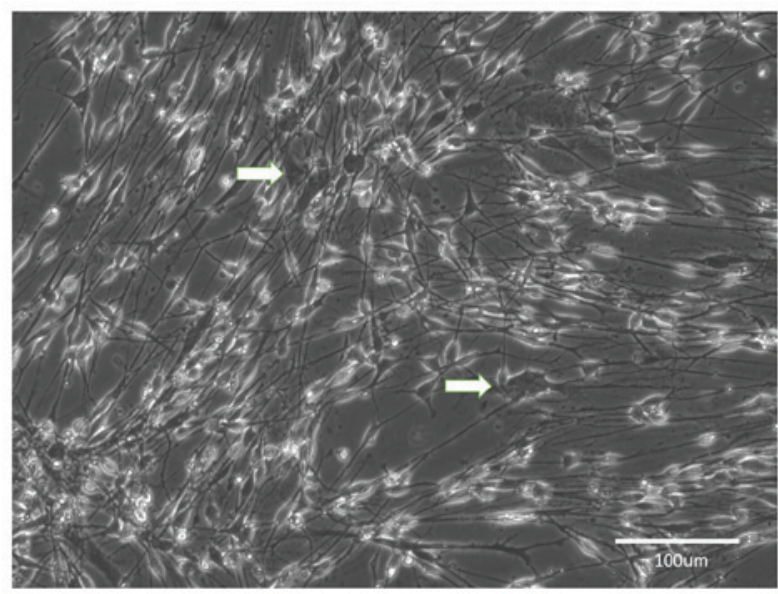

Figure 5. Group C. Cell culture after 21 days (scale bar, $100 \mu \mathrm{m}$ ). Most of the cells display a Schwann cell-like morphology. Only a few fibroblasts were observed (arrows).

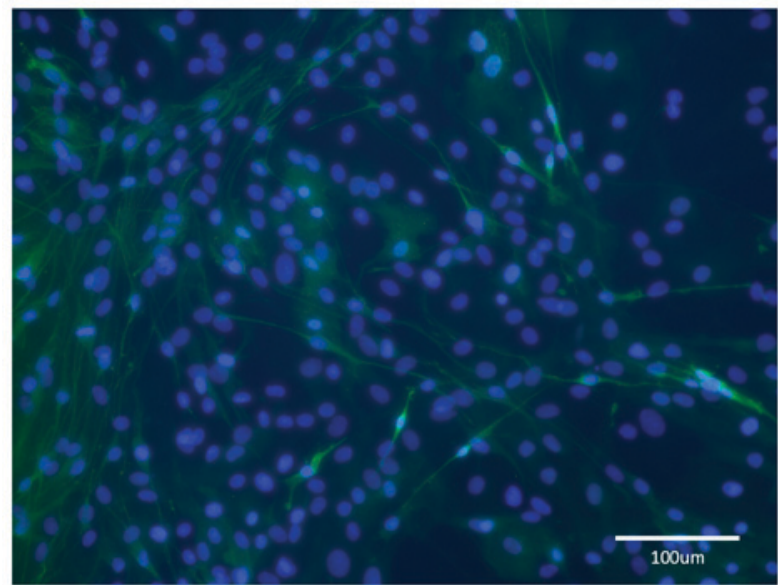

Figure 6. Group C. The cultured cells were fixed and immunostained after 21 days (scale bar, $100 \mu \mathrm{m}$ ). S100 (green) -immunopositive cytoplasm is present in cells with Schwann cell-like morphology. Nuclear staining was performed with DAPI (blue).

from peripheral nerves of newborn rats $(13,25)$. However, the purification and enrichment of SCs from adult peripheral nerve tissue is much more challenging. The problems associated with adult SC cultivation include i) the low yield of viable cells from the nerve tissue, ii) their poor proliferation capacity and iii) progressive overgrowth by the contaminating fibroblasts.

A study published by Kaewkhaw et al (26) appears to solve these problems by using a special medium containing D-valine. However, in all previosly used protocols, predegeneration of peripheral nerves is utilized $(15,18-20)$. The present study was based on the predegeneration protocol by Morissey et al (15) and its later modifications. The reasons for this decision were twofold: i) Problems with the availability of the medium (DMEM with D-valine) at the time of the experiments and ii) in the past, the predegeneration protocols were repeatedly used for producing SCs for transplantation experiments. It was documented that, even after several passages, the cultivated SCs maintain their ability to myelinize the regenerating axons. The known conditions were used in order 


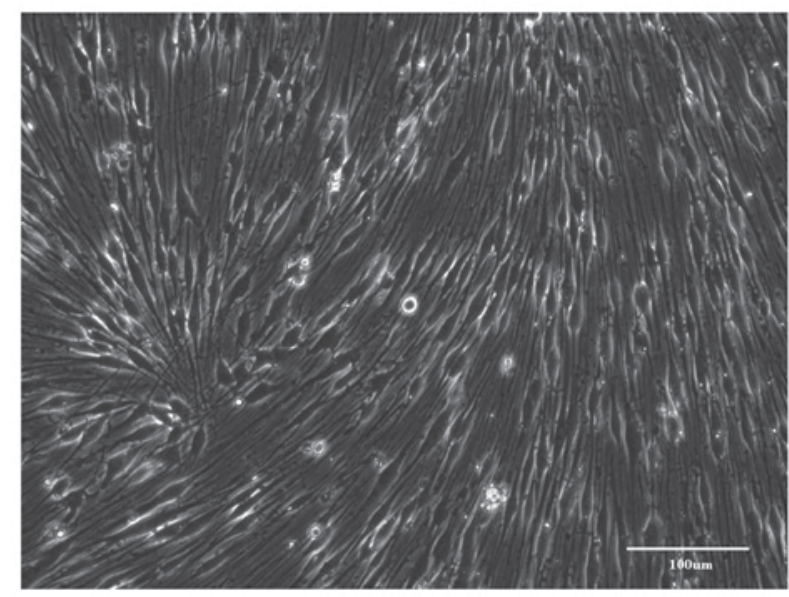

Figure 7. Group D. Proliferating Schwann cells after 14 days in culture form a monolayer (scale bar, $100 \mu \mathrm{m}$ ). Cells displayed a tendency to parallelize their processeses in the same direction and form typical waves-like structures.

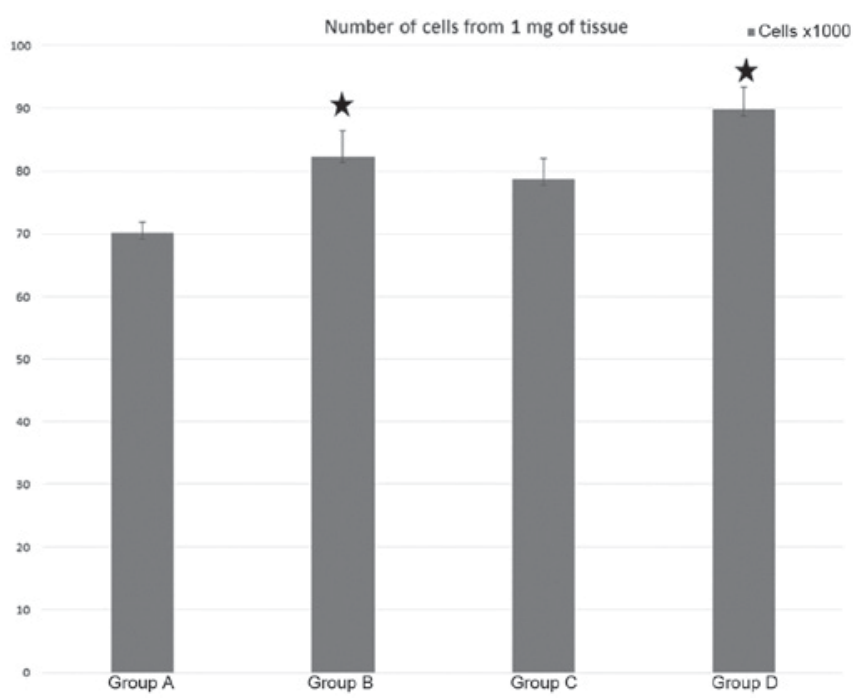

Figure 8. Numbers of cells after dissociation obtained from $1 \mathrm{mg}$ of tissue Group A (no predegeneration) served as a control and was compared with groups $\mathrm{B}-\mathrm{D} .{ }^{\star} \mathrm{P}<0.05$ vs. group $\mathrm{A}$.

to ensure that functional SCs were obtained in the subsequent transplantation experiments.

The predegeneration brings SCs into a mitotically activated state and enriches the cultures in favor of SCs as compared to fibroblasts. Initially, predegeneration in vivo was tested by transecting the peripheral nerve and leaving it in vivo for up to 21 days prior to harvesting and subsequent dissociation (19). From a clinical perspective, the in vivo predegeneration is questionable, as it requires two consequent surgical interventions. In addition, the degenerating nerves are also colonized by non-glial cells, particularly activated macrophages that may interfere with the subsequent cultivation. For these reasons, it is advisable to use in vitro predegeneration, which imitates Wallerian degeneration under controlled conditions. In previous studies, various durations of predegeneration have been used, varying between 1 and 5 weeks $(12,15,27,28)$. In an original study by Morrissey et al (15), a long predegeneration time (4-5 weeks) was advised. However, several other studies

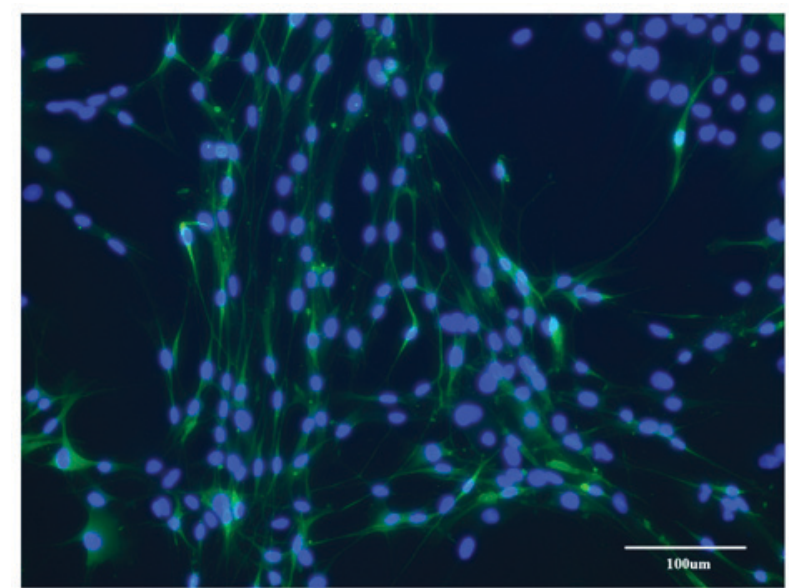

Figure 9. Group D. Immunostained Schwann cell culture after 21 days (scale bar, $100 \mu \mathrm{m})$. Green color, S100 immunostaining; blue color, DAPI.

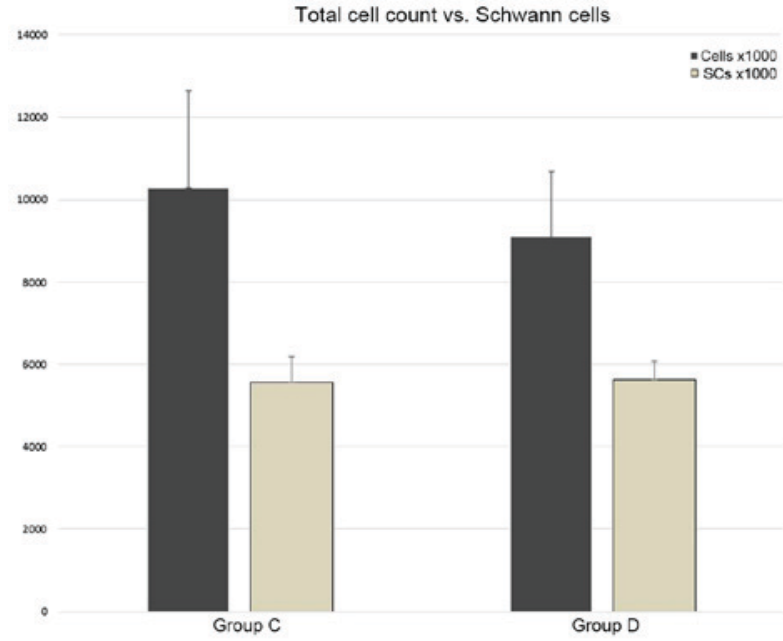

Figure 10. Quantified numbers of cells after 21 days of cultivation. Comparison of the total number of cells vs. the number of SCs in groups C and D. SCs, Schwann cells.

have used much shorter predegeneration periods (18-20,28). Kraus et al (27) have assessed the effect of different predegeneration times on the cell count and purity of SC cultures. They performed predegeneration for 2, 7 and 14 days, and reported the highest SCs counts after 7 days of predegeneration. Similar results have been reported by Niapour et al (12) in a study with canine SCs. The initial experiments on SC cultivation in our group used shorter predegeneration times (up to 2 weeks), but resulted in rapid debasement of the culture by proliferating fibroblasts. It was therefore decided to systematically assess the effect of different predegeneration times on the quality of SC cultures.

In the present study, minimal differences were observed between the cultures prepared immediately after harvesting and after 2 weeks of predegeneration. In those cases, the cultures were rapidly overgrown by fibroblasts. In experimental groups A and B, the initial number of SCs, as identified by their typical morphology, was low. In addition, the rapidly growing population of fibroblasts had a detrimental effect on the proliferation of the SC population and thus, the SCs were 
almost completely eliminated from the cultures at the end of the cultivation period. In group $\mathrm{C}$, the cultures contained either a highly enriched population of SCs, or in certain wells, the fibroblast population prevailed. In group D, all of the wells contained cultures highly enriched with SCs.

These results indicate a gradual improvement of SC purity associated with long-term predegeneration. It is known that denervated SCs produce and secrete large amounts of neurotrophic factors after nerve injury (29). Upregulation of neurotrophic factors and their receptors may contribute to the proliferation of SCs during Wallerian degeneration, and also in primary cultures of pre-activated SCs. After transection of the peripheral nerve, marked changes in the levels of tumor necrosis factor (TNF)- $\alpha$, interferon $\gamma$ and interleukin-1 have been described after 7,14 and 35 days (30). Interleukin-1 enhances fibroblast growth (31) and tumor necrosis factor $\alpha$ is a chemotactic agent for fibroblasts (32).

In the developing in vitro cultures in the present study, these two populations (fibroblasts and SCs) were influencing each other during the proliferation phase. When the fibroblasts prevailed, they had a tendency to form a monolayer and almost completely eliminate SCs from the culture. When the SCs prevailed, they proliferated rapidly and formed a near-pure culture that was intermingled with solitary fibroblasts. However, with prolonged cultivation, the number of fibroblasts in the SC culture slowly increased. For this reason, in the 21 day-old cultures of the present study, the percentage of SCs $(54,02 \%$ in group C and $61,95 \%$ in group D) was lower than that reported in previous studies $(12,15,17-19,21,26,27)$.

The present results are consistent with those reported by Morissey et al (15), who recommend 4-5 weeks of predegeneration and appear to contradict the previous observations indicating that the predegeneration periods of $>7$ days are associated with worse cell counts and purity of the final culture (26). This difference may be influenced by the fact that animals older than 3 months were used in the present study. It is known that in newborn animals, the process of Wallerian degeneration is faster (33) and that the recovery of the transected nerves is slower in older animals (34).

In conclusion, the major result of the present study is that when preparing pimary SC cultures from adult rats, long predegeneration periods are critical in order to produce enriched SC cultures. In our hands, the prolonged predegeneration only modestly improved cell yields after dissociation. It may thus be hypothesized that the prolonged predegeneration minimizes the proliferation activity of fibroblasts in favor of SCs.

The primary goal of the present study was to produce a population of cultured SCs that may be further used for autotransplantation studies. While this has been achieved, it was also revealed that the populations of cultivated SCs undego dynamic changes. In further studies, the proliferation index of the SCs in culture will be assessed and optimal periods of cultivation with respect to the purity of the SC population will be determined.

\section{Acknowledgements}

Preliminary results have been presented as a poster at FENS meeting, Copenhagen 2016.

\section{Funding}

The present study was supported by grants APVV-14-0847 (Slovak research and development agency) and VEGA 2/0145/16 (Scientific Grant Agency, Ministry of education, science, research and sport of the Slovak republic and Slovak Academy of Sciences).

\section{Availability of data and materials}

The analyzed data sets generated during the study are available from the corresponding author on reasonable request.

\section{Authors' contributions}

PT was responsible for the concept and design of the study, and contributed to the literature search. LS contributed to the literature search, analysed the data and reviewed the manuscript. IV contributed to the design of the study and the literature search. The final version of the manuscript has been read and approved by all authors, and each author believes that the manuscript represents honest work.

\section{Ethical approval and consent to participate}

This study was performed with the approval and according to the guidelines of the Institutional Animal Care and Use Committee of the Slovak Academy of Sciences (Košice, Slovakia) and with the European Communities Council Directive (2010/63/EU) regarding the use of animals in research and Slovak Law for Animal Protection 377/2012 and 436/2012.

\section{Consent for publication}

Not applicable.

\section{Competing interests}

The authors declare that they have no competing interests.

\section{References}

1. Noble J, Munro CA, Prasad VS and Midha R: Analysis of upper and lower extremity peripheral nerve injuries in a population of patients with multiple injuries. J Trauma 45: 116-122, 1998.

2. Robinson LR: Traumatic injury to peripheral nerves. Muscle Nerve 23: 863-873, 2000.

3. Evans-Jones G, Kay SP, Weindling AM, Cranny G, Ward A, Bradshaw A and Hernon C: Congenital brachial palsy: Incidence, causes, and outcome in the United Kingdom and Republic of Ireland. Arch Dis Child Fetal Neonatal Ed 88: F185-F189, 2003.

4. Johnson EO and Soucacos PN: Nerve repair: Experimental and clinical evaluation of biodegradable artificial nerve guides. Injury 3 (39 Suppl): S30-S36, 2008.

5. Evans GR: Peripheral nerve injury: A review and approach to tissue engineered constructs. Anat Rec 263: 396-404, 2001.

6. Kehoe S, Zhang XF and Boyd D: FDA approved guidance conduits and wraps for peripheral nerve injury: A review of materials and efficacy. Injury 43: 553-572, 2012.

7. Guénard V, Kleitman N, Morrissey TK, Bunge RP and Aebisher P: Syngeneic Schwann cells derived from adult nerves seeded in semipermeable guidance channels enhance peripheral nerve regeneration. J Neurosci 12: 3310-3320, 1992. 
8. Calancie B, Madsen PW, Wood P, Marcillo AE, Levi AD and Bunge RP: A guidance channel seeded with autologous Schwann cells for repair of cauda equina injury in a primate model. J Spinal Cord Med 32: 379-388, 2009.

9. Kim HA, Mindos T and Parkinson DB: Plastic fantastic: Schwann cells and repair of the peripheral nervous system. Stem Cells Transl Med 2: 553-557, 2013.

10. Hall SM: Regeneration in cellular and acellular autografts in the peripheral nervous system. Neuropathol Appl Neurobiol 12 27-46, 1986.

11. Kocsis JD and Waxman SG: Schwann cells and their precursors for repair of central nervous system myelin. Brain 130 1978-1980, 2007.

12. Niapour N, Mohammadi-Ghalehbin B, Golmohammadi MG Amani M, Salehi H and Niapour A: Efficacy of optimized in vitro predegeneration period on the cell count and purity of canine Schwann cell culture. Iran J Basic Med Sci 18: 307-311, 2015.

13. Brockes JP, Fields KL and Raff MC: Studies on cultured rat Schwann cells. I. Establishment of purified populations from cultures of peripheral nerve. Brain Res 165: 105-118, 1979.

14. Timmer M, Robben S, Müller-Ostermeyer F, Nikkah G and Grothe C: Axonal regeneration across long gaps in silicone chambers filled with Schwann cells overexpressing high molecular weight FGF-2. Cell Transplant 12: 265-277, 2003.

15. Morissey TK, Kleitman N and Bunge RP: Isolation and functional characterization of Schwann cells derived from adult peripheral nerve. J Neurosci 11: 2433-2442, 1991.

16. Rutkowski JL, Tennekoon GI and McGilicuddy JE: Selective culture of mitotically active human Schwann cells from adult sural nerves. Ann Neurol 31: 580-586, 1992.

17. Jirsová K, Soddar P, Mandys V and Bär PR: Cold jet: A method to obtain pure Schwann cell cultures without the need for cytotoxic, apoptosis-inducing drug treatment. J Neurosci Methods 78 133-137, 1997.

18. Keilhoff G, Fansa H, Schneider W and Wolf G: In vivo predegeneration of peripheral nerves: An effective technique to obtain activated Schwann cells for nerve conduits. J Neurosci Methods 89: 17-24, 1999.

19. Komiyama T, Nakao Y, Toyama Y, Asou H, Vacanti CA and Vacanti MP: A novel technique to isolate adult Schwann cells for an artificial nerve conduit. J Neurosci Methods 122: 195-200, 2003.

20. Verdú E, Rodríguez FJ, Gudiño-Cabrera G, Nieto-Sampedro M and Navarro X: Expansion of adult Schwann cells from mouse predegenerated peripheral nerves. J Neurosci Methods 99: 111-117, 2000.

21. Haastert-Talini K: Culture and proliferation of highly purified adult Schwann cells from rat, dog, and man. Methods Mol Biol 843: 189-200, 2012.
22. David S and Aguayo AJ: Axonal elongation into peripheral nervous system 'bridges' after central nervous system injury in adult rats. Science 214: 931-933, 1981.

23. Gu X, Ding F and Williams DF: Neural tissue engineering options for peripheral nerve regeneration. Biomaterials 35 : 6143-6156, 2014

24. Faroni A, Smith RJ, Lu L and Reid AJ: Human Schwann-like cells derived from adipose-derived mesenchymal stem cells rapidly de-differentiate in the absence of stimulating medium. Eur J Neurosci 43: 417-430, 2016.

25. Wood PM: Separation of functional Schwann cells and neurons from normal peripheral nerve tissue. Brain Res 115: 361-375, 1976.

26. Kaewkhaw R, Scutt AM and Haycock JW: Integrated culture and purification of rat Schwann cells from freshly isolated adult tissue. Nat Protoc 7: 1996-2004, 2012.

27. Kraus A, Täger J, Kohler K, Manoli T, Haerle M, Werdin F, Hoffman J, Schaller HE and Sinis N: Efficacy of various durations of in vitro predegeneration on the cell count and purity of rat Schwann-cell cultures. J Neurotrauma 27: 197-203, 2010.

28. Mauritz C, Grothe C and Haastert K: Comparative study of cell culture and purification methods to obtain highly enriched cultures of proliferating adult rat Schwann cells. J Neurosci Res 77: 453-461, 2004.

29. Fawcett JW and Keynes RJ: Peripheral nerve regeneration. Annu Rev Neurosci 13: 43-60, 1990.

30. Ruohonen S, Khademi M, Jagodic M, Taskinen HS, Olsson T and Röyttä M: Cytokine responses during chronic denervation. J Neuroinflammation 2: 26, 2005.

31. Singh JP, Adams LD and Bonin PD: Mode of fibroblast growth enhancement by human interleukin-1. J Cell Biol 106: 813-819, 1988.

32. Postlethwaite AE and Seyer JM: Stimulation of fibroblast chemotaxis by human recombinant tumor necrosis factor alpha (TNF-alpha) and a synthetic TNF-alpha 31-68 peptide. J Exp Med 172: 1749-1756, 1990.

33. Vyas A, Li Z, Aspalter M, Feiner J, Hoke A, Zhou C, O'Daly A, Abdullah M, Rohde C and Brushart TM: An in vitro model of adult mammalian nerve repair. Exp Neurol 223: 112-118, 2010.

34. Choi SJ, Harii K, Lee MJ, Furuya F and Ueda K: Electrophysiological, morphological, and morphometric effects of aging on nerve regeneration in rats. Scand J Plast Reconstr Surg Hand Surg 29: 133-140, 1995.

This work is licensed under a Creative Commons Attribution-NonCommercial-NoDerivatives 4.0 International (CC BY-NC-ND 4.0) License. 